\title{
$A b$ initio theory of magnetization induced by light absorption in ferromagnets
}

\author{
Philippe Scheid, ${ }^{1,2, *}$ Gregory Malinowski, ${ }^{2}$ Stéphane Mangin $\odot,{ }^{2}$ and Sébastien Lebègue ${ }^{1}$ \\ ${ }^{1}$ Université de Lorraine, LPCT, CNRS, UMR 7019, BP 70239, 54506 Vandoeuvre-lès-Nancy Cedex, France \\ ${ }^{2}$ Université de Lorraine, IJL, CNRS, UMR 7198, BP 70239, 54000 Nancy Cedex, France
}

(Received 6 June 2019; published 2 December 2019)

\begin{abstract}
We use density functional theory to quantitatively compute the effect of light absorption in ferromagnetic materials. We show that, in the presence of spin-orbit coupling, optically induced transitions do not conserve the magnetization and that a systematic induced demagnetization, whose magnitude depends on both the helicity of the light and the direction of the magnetization, is observed. Very differently from the inverse Faraday effect, this mechanism is due to the absorption of light and depends on the magnetic state of each atom, and therefore cannot be described by an effective optomagnetic field. Then, based on these results, we derive a set of parameters which can be used in micromagnetic simulations in order to account for light transition effects on the magnetization dynamics.
\end{abstract}

DOI: 10.1103/PhysRevB.100.214402

To face the continuing demand for large density and energy efficient data storage devices, the possibilities of magnetization manipulation without using a magnetic field are widely being investigated [1]. One of the promising candidates is the all-optical helicity-dependent switching (AO-HDS), as it allows the control of the magnetization state by only using circularly left $\left(\sigma^{+}\right)$or right $\left(\sigma^{-}\right)$polarized light pulses of a few tens of femtoseconds. AO-HDS has been observed in a wide range of materials such as $\mathrm{GdFeCo}$ ferrimagnetic alloys [2], rare earth-transition metal alloys and multilayers [3,4], and FePt $L 1_{0}$ granular media [5] which is considered promising for ultrahigh-density storage devices. This outstanding diversity of materials suggests a common underlying mechanism, although it remains debated.

To explain the AO-HDS, the two theoretical explanations usually invoked in the literature are the inverse Faraday effect (IFE) $[2,4-6]$ and a difference of absorption induced by the magnetic circular dichroism (MCD) $[7,8]$. While the IFE was first introduced to describe the influence of the presence of a circularly polarized light on the magnetic state of transparent media [9], Battiato et al. showed that, without any assumption on the nature of the material, light generates a static contribution at the second-order perturbation in the density matrix $[10,11]$, which they hold responsible for the IFE in lossy media. However, in this approach [10] the repopulation at the origin of the IFE does not grow linearly with time, as it would be the case for an absorption-related phenomenon, and it fades away after the perturbation has been switched off. This fact leaves a gap between the IFE and the mechanism involved in the irreversible change of magnetization leading to the AO-HDS phenomenon [12]. Then, using this formulation and density functional theory, Berritta et al. [13] computed the value of this contribution for different types of materials. Conversely, the second effect, due to MCD, relies on a difference of absorption inducing a different temperature depending on

\footnotetext{
*philippe.scheid@univ-lorraine.fr
}

the relative orientation of the magnetization and the helicity of the light. Through this thermal effect, the switching probability depends on the magnetization orientation, as shown by several parametrized models [7,8]. Moreover, its probabilistic and absorption-based nature is in agreement with the fact that the AO-HDS phenomenon is cumulative, i.e., it requires multiple pulses [4], as well as a large absorptivity of the compound.

In this paper, we demonstrate that another phenomenon occurs during the absorption of a circularly polarized light and induces a helicity-dependent magnetization in magnetic materials. Unlike the IFE which scales linearly with the intensity of the light, and therefore fades away with it, it is a dissipative phenomenon, whose contribution grows linearly both with the intensity of the light and with time. Furthermore, as this contribution is due to absorption, the angular momentum transferred from the light to the matter is preserved even after the light is gone, which makes it a good candidate in the explanation of the AO-HDS. Also, contrary to the statistical thermal effect discussed supra, this effect is purely optical.

To compute the rate of transition per unit of time from the state $\left|\psi_{n \vec{k}}\right\rangle$ to $\left|\psi_{n^{\prime} \cdot}\right\rangle$ induced by $\hat{W}$, the light-matter interaction Hamiltonian, we use the Fermi golden rule,

$$
\begin{aligned}
\Gamma_{n \vec{k} \rightarrow n^{\prime} \vec{k}}= & \frac{2 \pi}{\hbar}\left|\left\langle\psi_{n^{\prime} \vec{k}}|\hat{W}| \psi_{n \vec{k}}\right\rangle\right|^{2} f_{n, \vec{k}}\left(1-f_{n^{\prime}, \vec{k}}\right) \\
& \times\left[\delta\left(\varepsilon_{n^{\prime}, \vec{k}}-\varepsilon_{n, \vec{k}}-\hbar \omega\right)+\delta\left(\varepsilon_{n^{\prime}, \vec{k}}-\varepsilon_{n, \vec{k}}+\hbar \omega\right)\right],
\end{aligned}
$$

where $n$ is the band index, $\vec{k}$ a vector of the first Brillouin zone, $f_{n \vec{k}}$ and $\varepsilon_{n \vec{k}}$ are the occupation and energy of the orbital $\left|\psi_{n \vec{k}}\right\rangle$, and $\omega$ is the pulsation of the light, where $\varepsilon_{n \vec{k}}$ and $\left|\psi_{n \vec{k}}\right\rangle$ are obtained from fully noncollinear density functional theory calculations $[14,15]$. Then, the variation of the occupation number $f_{n \vec{k}}$ of the state $\left|\psi_{n \vec{k}}\right\rangle$ induced by the light writes as

$$
\frac{\partial f_{n, \vec{k}}}{\partial t}=\sum_{n^{\prime}}\left(\Gamma_{n^{\prime} \vec{k} \rightarrow n \vec{k}}-\Gamma_{n \vec{k} \rightarrow n^{\prime} \vec{k}}\right) .
$$


The coupling between the electrons and the electromagnetic field is expressed in the dipole approximation, i.e., with the Hamiltonian $\hat{W}=-\frac{q_{e}}{m_{e}}\|A\| \overrightarrow{\hat{p}} \cdot \vec{u}$, where $\|\vec{A}\|$ is the modulus of the potential vector of the light, $\overrightarrow{\hat{p}}$ the momentum operator acting on the wave functions of the electrons, and $\vec{u}$ is the polarization vector of the light. We only account for the vertical transition as at the considered photon energies, the wave vector of the light is small compared to the size of the Brillouin zone.

In the absence of spin-orbit coupling the Kohn-Sham orbitals are pure spin states. As $\hat{W}$ does not act on the spin part of the wave function, transitions between states with different spin parts are forbidden. However, $\hat{W}$ can, in the presence of spin-orbit coupling, induce transitions between electronic states having different, and even opposite, magnetizations. Indeed, in this case, $\left[\hat{H}, \hat{\sigma}_{z}\right] \neq 0$ and the Kohn-Sham orbitals are spinor states, e.g., they write as $\psi_{n \vec{k}}(\vec{r})=\left[a_{n \vec{k}}(\vec{r})|\uparrow\rangle+b_{n \vec{k}}(\vec{r})|\downarrow\rangle\right] e^{i \vec{k} \vec{r}}$. The magnetization of such a state can be defined as $\Uparrow$ (up) when $\left\langle\psi_{n \vec{k}} \mid \uparrow\right\rangle\left\langle\uparrow \mid \psi_{n \vec{k}}\right\rangle>\left\langle\psi_{n \vec{k}} \mid \downarrow\right\rangle\left\langle\downarrow \mid \psi_{n \vec{k}}\right\rangle$ and $\Downarrow$ (down) otherwise. The coupling of two such states by $\hat{W}$ is now proportional to $\left|\left\langle a_{n \vec{k}}^{\Uparrow}|\vec{p} \cdot \vec{u}| a_{n^{\prime} \vec{k}}^{\Downarrow}\right\rangle\right|^{2}+\left|\left\langle b_{n \vec{k}}^{\Uparrow}|\vec{p} \cdot \vec{u}| b_{n^{\prime} \vec{k}}^{\Downarrow}\right\rangle\right|^{2} \geqslant 0$, i.e., transitions between $\Uparrow$ and $\Downarrow$ states are not systematically forbidden.

Assuming that the orbital magnetization is quenched, as it is the case for all of the compounds studied here, the total magnetization writes as the sum of all the electron spinor magnetizations,

$$
\vec{M}=\mu_{B} \sum_{n} \frac{1}{\Omega_{\mathrm{BZ}}} \int_{\mathrm{BZ}}\left\langle\psi_{n \vec{k}}|\overrightarrow{\hat{\sigma}}| \psi_{n \vec{k}}\right\rangle f_{n \vec{k}} d \vec{k},
$$

where the integral is performed over the first Brillouin zone. From Eq. (3), the variation of the magnetization with respect to time due to a change in occupation writes as

$$
\frac{\partial \vec{M}}{\partial t}=\mu_{B} \sum_{n} \frac{1}{\Omega_{\mathrm{BZ}}} \int_{\mathrm{BZ}}\left\langle\psi_{n \vec{k}}|\overrightarrow{\hat{\sigma}}| \psi_{n \vec{k}}\right\rangle \frac{\partial f_{n \vec{k}}}{\partial t} d \vec{k},
$$

where we neglected the time dependence of the states. We now use Eqs. (2) and (4) to study the magnetization induced by circularly left and right polarized light, $\sigma^{+}=\frac{1}{\sqrt{2}}(1, i)^{t}$ and $\sigma^{-}=\frac{1}{\sqrt{2}}(1,-i)^{t}$, in the magnetic compounds fcc $\mathrm{Ni}$, bcc Fe, hcp Co, and FePt $L 1_{0}$. The magnitude of the atomic magnetization is $0.6 \mu_{B}$ in fcc $\mathrm{Ni}, 2.2 \mu_{B}$ in bcc Fe, $1.6 \mu_{B}$ in hcp Co, and $[3.1,0.3] \mu_{B}$ for $[\mathrm{Fe}, \mathrm{Pt}]$ in FePt $L 1_{0}$. The direction of the magnetization of every atom is along the easy axis $z$, and is positive for every compound, which is also set to be the direction of the propagation of the light. The electronic eigenstates, eigenvalues, and momentum matrix elements have been extracted from the full-potential, noncollinear linearized augmented plane-wave (LAPW) ELK code [16]. From these, in our separate PYTHON code, we implemented the computation of all the equations presented here. A Lorentzian with a broadening of $0.27 \mathrm{eV}$ has been used to smear the Dirac deltas.

Figure 1 shows the calculated induced magnetization for each polarization of the light, $\frac{\partial M^{\sigma^{+}, \sigma^{-}}}{\partial t}$, as well as $\frac{\partial M^{\sigma^{+}}}{\partial t}-\frac{\partial M^{\sigma^{-}}}{\partial t}$ magnified by a factor 10 . For both polarizations and in each

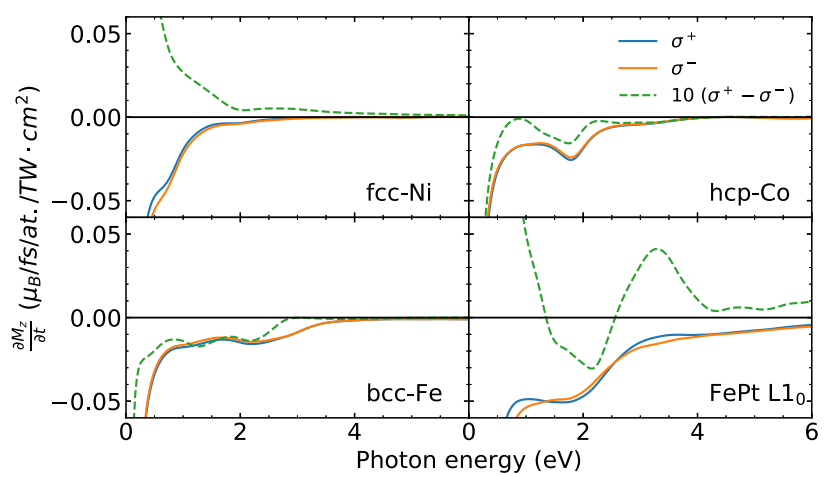

FIG. 1. Continuous lines: Variation of the magnetization with respect to time induced by optical transitions in fcc $\mathrm{Ni}$, hcp Co, bcc $\mathrm{Fe}$, and $\mathrm{FePt} L 1_{0}$ for circularly left and right polarized light, $\sigma^{+}$and $\sigma^{-}$. Dashed line: Difference of the variation of the magnetization induced by $\sigma^{+}$and $\sigma^{-}$polarized light with respect to time scaled by a factor 10 .

compound, the induced magnetization is opposite to the existing one. Figure 1 also exhibits a dependence of the induced magnetization on the polarization of the light, which is best seen by looking at the difference of the induced magnetization by $\sigma^{+}$and $\sigma^{-}$polarized light. Interestingly enough, for a given helicity the favored direction depends on the material, and for the same compound it can also depend on the photon energy, as evidenced in the case of FePt $L 1_{0}$. The spin-orbit coupling also seems to play an important role in the difference of induced magnetization, as in FePt $L 1_{0}$ it is larger than in bcc Fe. Also, this effect strongly weakens as the photon energy increases and becomes almost unnoticeable above $4 \mathrm{eV}$. This phenomenon may play a primary role in the AO-HDS, as each helicity tends to favor an opposite magnetization state.

To better understand the origin of this induced demagnetization, we define a magnetization resolved joint density of state,

$$
\begin{aligned}
& \operatorname{JDOS}_{\alpha^{\prime} \alpha}(\omega) \\
& =\sum_{n} \sum_{n^{\prime} \neq n} \frac{1}{\Omega_{\mathrm{BZ}}} \int_{\mathrm{BZ}}\left[f_{n \vec{k}}\left(1-f_{n^{\prime} \vec{k}}\right) \mathcal{H}\left(\alpha M_{z, n \vec{k}}\right) \mathcal{H}\left(\alpha^{\prime} M_{z, n^{\prime} \vec{k}}\right)\right. \\
& \left.\quad \times\left[\delta\left(\varepsilon_{n^{\prime} \vec{k}}-\varepsilon_{n \vec{k}}-\hbar \omega\right)-\delta\left(\varepsilon_{n^{\prime} \vec{k}}-\varepsilon_{n \vec{k}}+\hbar \omega\right)\right]\right] d \vec{k},
\end{aligned}
$$

where $M_{z, n \vec{k}}=\mu_{B}\left\langle\psi_{n \vec{k}}\left|\hat{\sigma}_{z}\right| \psi_{n \vec{k}}\right\rangle$ is the value of the magnetization of a Kohn-Sham orbital $\left|\psi_{n \vec{k}}\right\rangle$, projected along the easy axis, $\mathcal{H}$ is the Heaviside distribution, and $\alpha$ and $\alpha^{\prime}$ can be + or - to select $\Uparrow$ or $\Downarrow$ states, allowing us to break down all the possible transitions into $\Uparrow$ to $\Uparrow, \Uparrow$ to $\Downarrow, \Downarrow$ to $\Uparrow$, and $\Downarrow$ to $\Downarrow$. Figure 2 shows the calculated joint density of states for every compound studied. In each case, transitions from $\Uparrow$ states to $\Downarrow$ states are dominant and the curves present a peak located at the value of the exchange splitting, which is also noticeable in Fig. 1. It is well explained by the fact that because of the exchange splitting, the states having energies below $\epsilon_{F}$, the Fermi energy, are mainly $\Uparrow$, while a greater number of $\Downarrow$ states are available above $\epsilon_{F}$. For the same reason, the number of $\Uparrow$ states above $\epsilon_{F}$ is very low in comparison with the number of $\Downarrow$ states. This imbalance between the number of available 


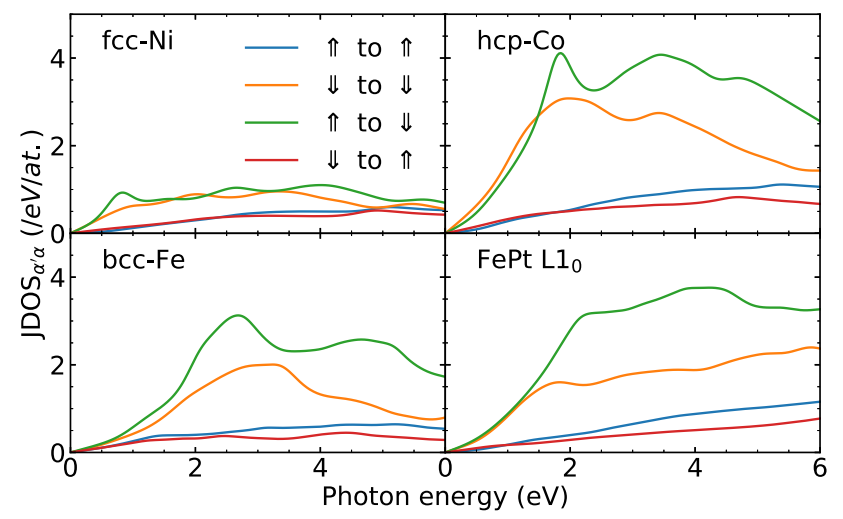

FIG. 2. Joint density of states of fcc $\mathrm{Ni}$, hcp Co, bcc Fe, and FePt $L 1_{0}$ broken down into the different initial and final magnetic states involved, as defined in Eq. (5).

transitions from $\Uparrow$ to $\Downarrow$ versus $\Downarrow$ to $\Uparrow$ and the fact that this type of excitation is made possible owing to the presence of spin-orbit coupling explains the systematic demagnetization.

Comparing bcc Fe with FePt $L 1_{0}$ allows us to understand the role of the spin mixing induced by the spin-orbit coupling. Indeed, whereas the JDOS ${ }_{\alpha^{\prime} \alpha}$ of bcc Fe and FePt $L 1_{0}$ are similar at $\approx 2 \mathrm{eV}$, meaning that the same imbalance of available transitions from $\Uparrow$ to $\Downarrow$ states and $\Downarrow$ to $\Uparrow$ states exists, the demagnetization induced in FePt $L 1_{0}$ is larger than in bcc Fe. This is explained by the increase of spin-orbit coupling produced by the addition of the Pt atom, which favors the spin mixing and allows more transitions from $\Uparrow$ states to $\Downarrow$ states.

As the mechanism of AO-HDS may rely on the propagation of domain walls [17], we generalize the previous results for some directions of the magnetization which are not collinear to the direction of propagation of the light. As sketched in Fig. 3, we rotate the magnetic moments of an angle $\theta$ varying from 0 to $\pi$ with respect to the direction of the light propagation in the plane (100) of the conventional cells of hcp Co, bcc Fe, and FePt $L 1_{0}$ and (110) of the conventional cell of fcc Ni. For every angle, we find that $\frac{\partial \vec{M}^{\sigma^{ \pm}}}{\partial t}$ is anticollinear to $\vec{M}$. Therefore, the effect of the light on the magnetization can be reduced to its action on the modulus

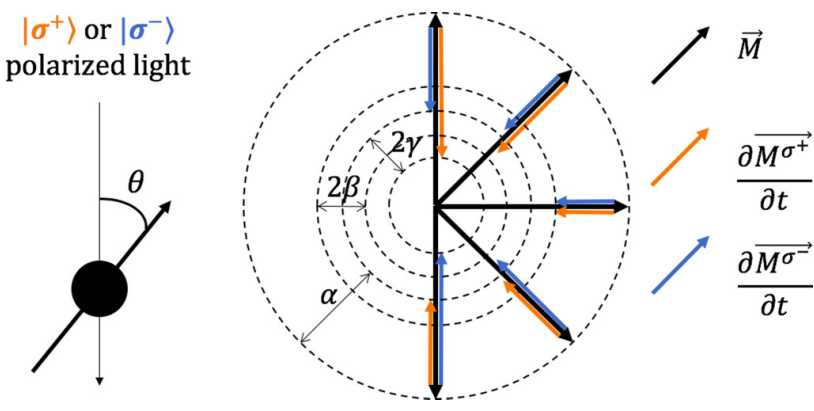

FIG. 3. Sketch of the induced magnetization produced by $\sigma^{+}$and $\sigma^{-}$polarized light per unit of time for different orientations of the magnetic moments with respect to the direction of propagation of the light. The parameters $\alpha, \beta$, and $\gamma$ of the fitting law defined by Eq. (6) are represented.

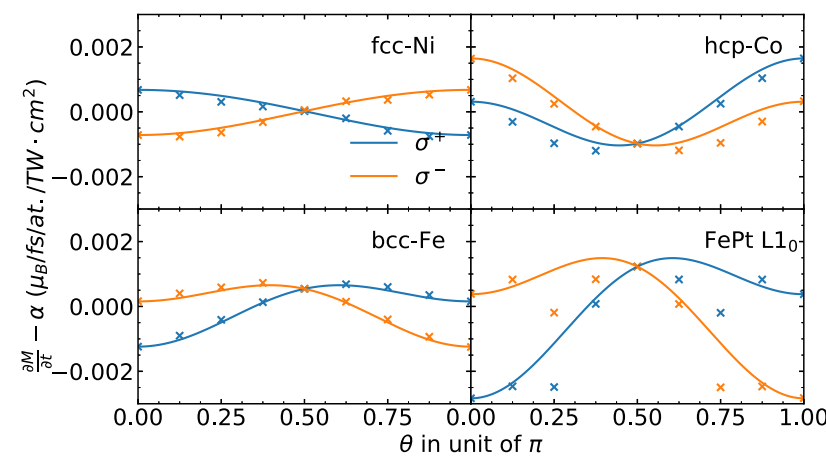

FIG. 4. Markers: Angle-dependent part of the magnetization induced by $\sigma^{+}$and $\sigma^{-}$polarized photons of $1.55 \mathrm{eV}$ per unit of time for different values of $\theta$, the angle between the light propagation axis and the magnetization. Continuous lines: Fit using Eq. (6) and the values of $\alpha, \beta$, and $\gamma$ given in Table I.

of the magnetization. These considerations are depicted in Fig. 3. Such a behavior strongly differs from the usual way micromagnetic simulations deal with the action of a polarized light on the magnetization, in which the induced optomagnetic field by the IFE is along the direction of the light propagation axis $[6,8]$.

To make our results valuable for future investigations of the AO-HDS using micromagnetic simulations $[6,8]$, as well as to understand the different phenomena taking place during the absorption, we fit our results with the law

$$
\frac{\partial M^{\sigma^{ \pm}}}{\partial t}=\alpha+\beta \cos (2 \theta) \pm \gamma \cos (\theta)
$$

where $\frac{\partial M^{\sigma^{ \pm}}}{\partial t}$ is the component of the induced magnetization per unit of time along the direction of the magnetization and $\alpha, \beta$, and $\gamma$ are the fitting parameters which may be used to improve atomistic micromagnetic calculations in order to account for the light-induced transition effects on the magnetization.

Each parameter has a physical meaning, allowing us to rationalize the magnetization behavior. Indeed, as evidenced in Fig. 4, where we fit the induced magnetization for different values of $\theta$, the action of the light on $\vec{M}$ can, in good approximation (especially in monoatomic compounds), be broken down into three effects. All of them are involving a different kind of angular momentum transfer induced by light. The first one, characterized by $\alpha$, is helicity and angle independent. It accounts for the systematic induced demagnetization, as its sign is negative for all the studied compounds. The second one, the magnitude and sign of which is given by $\beta$, is angle dependent, but helicity independent. It reflects the difference in the induced magnetization produced by the angle between the direction of propagation of the light and the direction of $\vec{M}$. Lastly, $\gamma$ represents the amplitude and sign of the helicity-dependent part of the light-induced magnetization. It vanishes when $\theta=\frac{\pi}{2}$ and has the opposite effect when $\vec{M}$ is parallel or antiparallel to the direction of propagation of the light.

Table I shows the values of $\alpha, \beta$, and $\gamma$ for fcc Ni, hcp $\mathrm{Co}$, bcc $\mathrm{Fe}$, and $\mathrm{FePt} L 1_{0}$ calculated at a photon energy 
TABLE I. Values of the parameters $\alpha, \beta$ and $\gamma$ corresponding to the law Eq. (6) for fcc $\mathrm{Ni}$, hep $\mathrm{Co}$, bcc $\mathrm{Fe}$, and FePt $L 1_{0}$ for photons of $1.55 \mathrm{eV}$.

\begin{tabular}{lcrr}
\hline \hline$\left(\mu_{B} / \mathrm{fs} /\right.$ at. $\left./ \mathrm{TW} \mathrm{cm}{ }^{2}\right)$ & $\alpha$ & \multicolumn{1}{c}{$\beta$} & \multicolumn{1}{c}{$\gamma$} \\
\hline fcc Ni & -0.00464 & -0.00006 & 0.00070 \\
hcp Co & -0.02086 & 0.00098 & -0.00066 \\
bcc Fe & -0.01233 & -0.00054 & -0.00070 \\
FePt $L 1_{0}$ & -0.04929 & -0.00061 & -0.00080 \\
\hline \hline
\end{tabular}

of $1.55 \mathrm{eV}$. It demonstrates that they are highly material dependent. Indeed, as previously pointed out, the values of $\alpha$, characterizing the light-induced demagnetization, are strongly influenced by the number of transition from $\Uparrow$ states to $\Downarrow$ states available, as well as the presence of large spin-orbit coupling which facilitates these transitions as brought by $\mathrm{Pt}$ atoms in FePt $L 1_{0}$. Furthermore, although the magnitudes of the effects represented by $\beta$ and $\gamma$ are roughly similar, their sign depends on the compound.

Evaluating the results in Table I for a fluence of $5 \mathrm{~mJ} / \mathrm{cm}^{2}[18]$ and a light propagating in the direction of the magnetization, we can compute that the induced demagnetization due to $\alpha$ is $3.9 \%$ of the magnetization in fcc $\mathrm{Ni}, 6.2 \%$ in hep Co, $2.9 \%$ in bcc Fe, and $15.3 \%$ in FePt $L 1_{0}$. As this demagnetization is due to optically induced transitions only, these results may explain the early phase of the demagnetization happening during the pulse through light-induced Stoner excitations. This contribution supplements the others, as, e.g., the ultrafast generation of magnons, whose roles are still heavily debated [19-24] in the explanation of ultrafast lightinduced demagnetization. However, unlike other mechanisms where the angular momentum has to be transferred to other baths, such as phonons through Elliott-Yafet scattering [18] or the ultrafast Einstein-de Haas effect [25], these Stoner excitations are directly induced by light and no other dissipation channel is required to allow a spin flip.

We now evaluate the difference of induced magnetization between both helicities, characterized by the parameter $\gamma$ in Table I. This difference amounts to $1.0 \%$ of the magnetization in fcc Ni, $0.4 \%$ in hep Co, $0.3 \%$ in bcc Fe, and $0.5 \%$ in FePt $L 1_{0}$. These rather small values are in agreement with the experimental fact that the AO-HDS is a cumulative effect, i.e., multiple pulses are needed to achieve a switching [5]. Moreover, unlike the inverse Faraday effect, the induced magnetization is preserved after the light is gone. As a consequence, much as the thermal effect induced by the MCD [8], this difference of induced magnetization can affect the dynamics on timescales longer than the pulse duration, and progressively favor one magnetic state over the other.

In conclusion, by considering the role of absorption, our results shed light on the all-optical helicity-dependent switching, as much as they raise questions on the nature of the underlying mechanism(s). Indeed, we demonstrated that lightinduced transitions affect the magnetization state of the ferromagnets fcc $\mathrm{Ni}$, hcp $\mathrm{Co}$, bcc $\mathrm{Fe}$, and FePt $L 1_{0}$. Due to the exchange splitting and the presence of spin-orbit coupling, the transition from majority spin states to minority spin states is dominant and, as a direct consequence, light always induces a demagnetization.

Furthermore, our $a b$ initio calculations showed that one helicity of the light tends to demagnetize more than the other. The favored magnetic state depends on both the material and the photon energy. Extending our computation to cases where the light does not propagate along the magnetization axis, we showed that an optomagnetic field, commonly used in micromagnetic calculation, cannot account for this effect, and we derived a set of parameters which can be used instead. As the inverse Faraday effect and the difference of absorption due to MCD, the phenomenon described here may play an essential role in the AO-HDS [4,5], in the HD domain wall motion [17], as well as in the HD demagnetization [26]. A further assessment of the magnitude of this effect using realtime time-dependent density functional theory [27] is planned. It will allow us to study the full response of the electronic system to a realistic circularly polarized light pulse.

The authors are very grateful to Peter Oppeneer, Eric Fullerton, and Jon Gorchon for the many insightful and stimulating discussions. P.S. was supported by the French PIA project Lorraine Université d'Excellence, Reference No. ANR-15-IDEX-0004.
[1] D. Sander, S. O. Valenzuela, D. Makarov, C. H. Marrows, E. E. Fullerton, P. Fischer, J. McCord, P. Vavassori, S. Mangin, P. Pirro, B. Hillebrands, A. D. Kent, T. Jungwirth, O. Gutfleisch, C. G. Kim, and A. Berger, J. Phys. D: Appl. Phys. 50, 363001 (2017).

[2] C. D. Stanciu, F. Hansteen, A. V. Kimel, A. Kirilyuk, A. Tsukamoto, A. Itoh, and T. Rasing, Phys. Rev. Lett. 99, 047601 (2007).

[3] S. Alebrand, M. Gottwald, M. Hehn, D. Steil, M. Cinchetti, D. Lacour, E. E. Fullerton, M. Aeschlimann, and S. Mangin, Appl. Phys. Lett. 101, 162408 (2012).

[4] S. Mangin, M. Gottwald, C. H. Lambert, D. Steil, V. Uhlî́, L. Pang, M. Hehn, S. Alebrand, M. Cinchetti, G. Malinowski, Y. Fainman, M. Aeschlimann, and E. E. Fullerton, Nat. Mater. 13, 286 (2014).
[5] C. H. Lambert, S. Mangin, B. S. S. Varaprasad, Y. K. Takahashi, M. Hehn, M. Cinchetti, G. Malinowski, K. Hono, Y. Fainman, M. Aeschlimann, and E. E. Fullerton, Science 345, 1337 (2014).

[6] K. Vahaplar, A. M. Kalashnikova, A. V. Kimel, S. Gerlach, D. Hinzke, U. Nowak, R. Chantrell, A. Tsukamoto, A. Itoh, A. Kirilyuk, and T. Rasing, Phys. Rev. B 85, 104402 (2012).

[7] J. Gorchon, Y. Yang, and J. Bokor, Phys. Rev. B 94, 020409(R) (2016).

[8] M. O. Ellis, E. E. Fullerton, and R. W. Chantrell, Sci. Rep. 6, 30522 (2016).

[9] P. S. Pershan, J. P. Van Der Ziel, and L. D. Malmstrom, Phys. Rev. 143, 574 (1966).

[10] M. Battiato, G. Barbalinardo, K. Carva, and P. M. Oppeneer, Phys. Rev. B 85, 045117 (2012). 
[11] M. Battiato, G. Barbalinardo, and P. M. Oppeneer, Phys. Rev. B 89, 014413 (2014).

[12] A. H. M. Reid, A. V. Kimel, A. Kirilyuk, J. F. Gregg, and T. Rasing, Phys. Rev. B 81, 104404 (2010).

[13] M. Berritta, R. Mondal, K. Carva, and P. M. Oppeneer, Phys. Rev. Lett. 117, 137203 (2016).

[14] P. Hohenberg and W. Kohn, Phys. Rev. 136, B864 (1964).

[15] W. Kohn and L. J. Sham, Phys. Rev. 140, A1133 (1965).

[16] http://elk.sourceforge.net/.

[17] Y. Quessab, R. Medapalli, M. S. El Hadri, M. Hehn, G. Malinowski, E. E. Fullerton, and S. Mangin, Phys. Rev. B 97, 054419 (2018).

[18] B. Koopmans, G. Malinowski, F. Dalla Longa, D. Steiauf, M. Fähnle, T. Roth, M. Cinchetti, and M. Aeschlimann, Nat. Mater. 9, 259 (2010).

[19] E. Beaurepaire, J. C. Merle, A. Daunois, and J. Y. Bigot, Phys. Rev. Lett. 76, 4250 (1996).

[20] G. P. Zhang and W. Hübner, Phys. Rev. Lett. 85, 3025 (2000).

[21] K. Krieger, J. K. Dewhurst, P. Elliott, S. Sharma, and E. K. Gross, J. Chem. Theory Comput. 11, 4870 (2015).
[22] E. Turgut, D. Zusin, D. Legut, K. Carva, R. Knut, J. M. Shaw, C. Chen, Z. Tao, H. T. Nembach, T. J. Silva, S. Mathias, M. Aeschlimann, P. M. Oppeneer, H. C. Kapteyn, M. M. Murnane, and P. Grychtol, Phys. Rev. B 94, 220408(R) (2016).

[23] S. Eich, M. Plötzing, M. Rollinger, S. Emmerich, R. Adam, C. Chen, H. C. Kapteyn, M. M. Murnane, L. Plucinski, D. Steil, B Stadtmüller, M. Cinchetti, M. Aeschlimann, C. M. Schneider, and S. Mathias, Sci. Adv. 3, e1602094 (2017).

[24] P. Tengdin, W. You, C. Chen, X. Shi, D. Zusin, Y. Zhang, C. Gentry, A. Blonsky, M. Keller, P. M. Oppeneer, H. C. Kapteyn, Z. Tao, and M. M. Murnane, Sci. Adv. 4, eaap9744 (2018).

[25] C. Dornes, Y. Acremann, M. Savoini, M. Kubli, M. J. Neugebauer, E. Abreu, L. Huber, G. Lantz, C. A. F. Vaz, H. Lemke, E. M. Bothschafter, M. Porer, V. Esposito, L. Rettig, M. Buzzi, A. Alberca, Y. W. Windsor, P. Beaud, U. Staub, D. Zhu, S. Song, J. M. Glownia, and S. L. Johnson, Nature (London) 565, 209 (2019).

[26] Y. Tsema, G. Kichin, O. Hellwig, V. Mehta, A. V. Kimel, A. Kirilyuk, and T. Rasing, Appl. Phys. Lett. 109, 072405 (2016).

[27] E. Runge and E. K. U. Gross, Phys. Rev. Lett. 52, 997 (1984). 